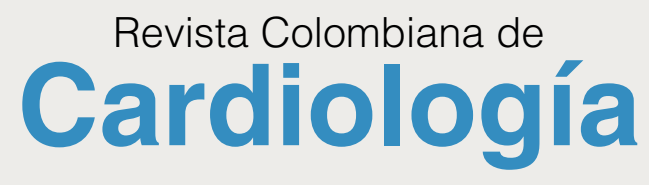

www.elsevier.es/revcolcar

HISTORIA DE LA CARDIOLOGÍA

\title{
Profesor Ramón Atalaya Varela: Padre de la Cardiología Colombiana
}

\section{Professor Ramon Atalaya Varela: Father of the Colombian Cardiology}

\author{
Alberto Barón Castañeda
}

Cardiología, Clínica de Marly S.A. Bogotá, Colombia

Recibido el 16 de junio de 2016; aceptado el 16 de junio de 2016

Disponible en Internet el 8 de julio de 2016

Vivimos en una época de grandes cambios, en la que la ciencia progresa a un ritmo vertiginoso con una cantidad inmensurable de investigaciones y publicaciones, muchas orientadas hacia el mejor entendimiento de las enfermedades y el desarrollo de nuevos tratamientos para aliviar o curar las enfermedades, otras a mejorar la calidad de vida o prolongar la duración de la misma. Cuando reflexionamos acerca de estos avances, un buen alivio de los síntomas en la mayoría de las enfermedades y curación de unas pocas, no podemos desconocer lo distantes que estamos del ideal de una curación total. Esta realidad nos lleva a vivir en el presente, proyectándonos hacia el futuro, reconociendo que el conocimiento actual es el resultado de un continuo de progresos que se han logrado a través del tiempo.

La Revista Colombiana de Cardiología es una herramienta que puede transmitir el conocimiento a las nuevas generaciones y puede ser útil para recordar la importancia de la historia.

El objetivo de este breve artículo es hacer un homenaje a un importante colega que nos antecedió y dejó un legado de conocimientos y desarrollo a la cardiología colombiana.

El doctor Ramón Atalaya Varela nació el 9 de octubre de 1902 en Bogotá, hijo de Francisco Atalaya Camacho e Ismenia Varela Santos. Se casó con doña Blanca Echevarría Toro, de cuyo matrimonio nacieron Mariluz, Juan Ramón,

Correo electrónico: albertobaronc@gmail.com
Clara Inés, José Luis Felipe, María Elena Isabel y Alberto Javier.

Estudió en la Facultad de Medicina de la Universidad Nacional, donde se graduó el 15 de noviembre de1928. Su tesis llevó como título: "Algunas investigaciones sobre la tensión arterial'. Fue ganador de la Beca de la Facultad de Medicina de la Universidad Nacional de Colombia, lo que le permitió viajar a Francia después de recibir su grado. En la Facultad de Medicina de París se especializó en Cardiología. En el Hospital de Paris fue alumno de importantes personalidades de la cardiología como Louis Henri Vaquez, Charles Laubry, C. Lian, Clere, René Lutembacher y Geraudel. Fue compañero del profesor Ignacio Chávez, de México, con quien mantuvo lazos de amistad. Los dos hicieron la promesa de crear un Instituto de Cardiología cuando cada uno regresara a su país. En 1930 cuando terminó la especialización, regresó a Bogotá.

Cuando retornó a Colombia trajo consigo el segundo electrocardiógrafo, después del importado por el Dr. Carlos Trujillo Gutiérrez, convirtiéndose en el primer electrocardiógrafo usado por un cardiólogo en el país. Con esto marcó un hito: dio inicio a este importante examen de diagnóstico.

Como pionero de la especialidad en Colombia en muy poco tiempo fundó los primeros servicios de Cardiología en la Clínica Marly, en el Hospital San Juan de Dios y en el Hospital San José, todos en Bogotá.

En 1934 fue nombrado Jefe de la Sección Médica del Instituto Nacional de Radium, precursor del actual Instituto 
Nacional de Cancerología, importante cargo que desempeñó hasta 1945. En forma paralela, fue presidente de la recién fundada Federación Médica Colombiana durante 1936 y 1937.

En 1949 fue nombrado Agregado Cultural de la embajada Colombiana en los Estados Unidos, motivo por el cual viajó a Washington, donde se vinculó con el hospital John Hopkins y conoció a profesionales sobresalientes.

Así mismo, se distinguió por su interés académico. Dirigió el "Laboratorio de Cardiología y Metabolismo Basal" del hospital San Juan de Dios, que fue la base para crear luego la Sección de Cardiología. Fue distinguido con el título de profesor titular, por concurso, de la Facultad de Medicina de la Universidad Nacional de Colombia a partir de 1939. "Extraía los síntomas del enfermo con su más recóndito significado y los transmitía a sus alumnos con tal fuerza y objetividad que estos veían, palpaban y auscultaban con los ojos, las manos y los oídos del profesor' '. En 1941 fue designado Director del Departamento de Cardiología y fue además Profesor Titular en la Pontificia Universidad Javeriana.

En 1944 fue admitido como miembro de Número de la Academia Nacional de Medicina; para esta ocasión presentó su trabajo "Arteriosis pulmonar solitaria"', significativa descripción de la hoy temida hipertensión pulmonar. En esa institución ocupó cargos como vocal de la Junta Directiva y fue su vicepresidente durante el período 1952 a 1954. Fue Miembro Correspondiente de las Academias de Medicina de Medellín y de Cartagena, Miembro Correspondiente de la Sociedad Mexicana de Cardiología y de la Sociedad Interamericana de Cardiología, donde actuó como miembro de su comité directivo, Miembro de la Sociedad Colombiana de Medicina Interna, perteneció a la Sociedad de Cirugía de Bogotá y fue Fundador y Jefe del departamento de Cardiología del Hospital San José de Bogotá.

Con la idea de organizar una "entidad que habrá de dedicarse al estudio de los problemas de la fisiología, patología y fisiopatología y terapéutica del sistema vascular en nuestro medio', el 18 de septiembre de 1943 fundó la Sociedad Colombiana de Cardiología. La primera Junta Directiva fue presidida por el profesor Atalaya y lo acompañaron los doctores Jorge Salcedo Salgar, Francisco Gnecco Mazo, Jorge E. Bernal Tirado, Gonzalo Montes Duque, Alejandro Palacios y José D. Rojas Franky. Con la ayuda del profesor J. Hernando Ordóñez, el doctor Atalaya organizó el Primer Congreso Colombiano de Cardiología, que se llevó a cabo en Bogotá, desde el 12 hasta 14 de octubre de 1956. En este histórico evento se presentaron temas novedosos como la angiocardiografía, la utilidad del balistocardiograma, cuerpos extraños en el corazón y temas de cirugía como injertos arteriales y comisurotomía aórtica. Desde entonces los congresos se han realizado con periodicidad bienal sin interrupción hasta la fecha.

El 1 de julio de 1966 fue distinguido con la "Orden de Boyacá"'.

Falleció en Bogotá el 28 de mayo de 1967.

La Sociedad Colombiana de Cardiología y Cirugía Cardiovascular, en reconocimiento al insigne profesor Atalaya, ha elegido su nombre para el premio al mejor trabajo de investigación otorgado por esta institución en cada congreso.

\section{Conflicto de intereses}

El autor declara no tener ningún conflicto de intereses

\section{Bibliografía recomendada}

Barón A. Apuntes sobre la Historia de la Sociedad Colombiana de Cardiología. Boletín Sociedad Colombiana de Cardiología. Bogotá; 2001.

Cáceres H, Cuéllar-Montoya Z. Academia Nacional de Medicina de Colombia. Sus miembros 1873-1997. Bogotá; 1998.

Garavito F. El corazón de oro. Primera edición. Bogotá; 1993.

Lozano Bautista G. Historia de la Cardiología en Colombia. Conferencia dictada en el XII Congreso Colombiano de Cardiología en Cúcuta, 28 de Noviembre de 1987. Acta Médica Colombiana 1989;14:41-7.

Lozano G. Historia de la Cardiología en Colombia. Acta Med Col. 1989;14:41-7

Reseña Histórica de la Sociedad Colombiana de Cardiología y Cirugía Cardiovascular. Disponible en: www.scc.org.co/ mision-vision-e.historia/ 